\title{
Usage of alkaline glucose for Synthesis Copper Nano particle on Polyester Fabric
}

\author{
Mohammad Mahdi Jolaei ${ }^{1}$, , Majid Montazer ${ }^{2}$, Abo-Saeed Rashidi' ${ }^{1}$, Mohammad Bameni \\ Moghadam $^{3}$ \\ ${ }^{1}$ Department of Textile Engineering, Faculty of Engineering, Science and Research Branch, Islamic \\ Azad University, Tehran, Iran. \\ ${ }^{2}$ Department of Textile Engineering, Faculty of Textile Engineering, Amirkabir University of \\ Technology, Tehran, Iran. \\ ${ }^{3}$ Department of Statistics, Faculty of Mathematics and Computer Science, Allameh Tabataba'i \\ University, Tehran, Iran.
}

\begin{abstract}
In this study, Nano copper from the chemical reaction between copper salt and sodium hydroxide was produced. Interestingly, simultaneous synthesis of Nano-copper chemical method and depositing Nano materials on the fabric's surface. The glucose is used as a stabilizer. An important feature of this study is, simplicity, availability, low cost and etc. The results create Nano-copper with a thickness of 30-40 nanometers on the surface of the fabric is shown. The treated fabrics could be used in diverse areas such as defense, aerospace, electronics, medical and health industries.
\end{abstract}

Keywords: synthesis, Nano plates, polyester fabric, copper, glucose, alkaline. 


\section{Introduction}

$\mathrm{U}$ se of metal Nanoparticles is currently highly regarded due to their useful properties. Using Nano metals on textile causes development of new properties and characteristics on the fabric that is produced as a result of metal characteristics. In the past two decades, considerable studies have focused on synthesis of metal Nanoparticles in order to find potential applications. Decrease in particle size leads to increase in surface to particles volume rate with high increase in catalyst features of metal Nanoparticles. Chemical catalysts can effectively improve performance and structures by multiple productions of particles and strong chemical bonds between the matrix and particles cause some structural strength and increase.[1, 2] Copper and its compounds have been studied for many years, not only for their antibacterial activity, but also for their low toxicity.[3-10] Several methods have been developed for preparing copper Nanoparticles, which include photo-catalytic reduction, chemical reduction, photo-chemical or radiation-chemical reduction, metallic wire explosion, sono-chemical, polyols, matrix chemistry, photo-reduction, reverse micelle based methods and even biological synthesized.[11] Among various metal particles, use of copper Nanoparticles has been highly considered due to their availability, low cost and optical, electrical, catalytic, mechanical, magnetic and conductive properties.[12]

In previous studies of Nano-metal materials such as copper have been synthesized by various methods such as prepared in an aqueous solution using sodium dodecyl sulfate as capping agents.[13, 14] A method for synthesizing copper Nanoparticles has utilized charge compensatory effect of ionic liquid BF4 in conjunction with ethylene glycol for providing electro-steric stabilization to copper Nanoparticles prepared from copper sulfate using hydrazine hydrate as a reducing agent.[15] With an additional coating of a hydrophilic polymer has been carried out using hydrazine hydrate $(\mathrm{HH})$ and sodium formaldehyde sulfoxylate (SFS) in an aqueous medium[16]. Metal Nanoparticles such as $\mathrm{Ag}$ and $\mathrm{Cu}$ are found to have antibacterial activity.[17, 18] Bactericidal effect of metal Nanoparticles has been attributed to their small size and high surface to volume ratio, which allow them to closely interact with microbial membranes, which is not merely due to release of metal ions in solutions $[18,19]$.

Cyclodextrin is a family of cyclic oligosaccharides, which is made of attached units of glucopyranosyl $\alpha-(1,4)$. The Cyclodextrin (CD) oligosaccharides are made from 6 to 8 units D-glucose $(\alpha-C D=6, \beta$ $\mathrm{CD}=7, \quad \gamma-\mathrm{CD}=8)$. Cyclodextrin chemistry and oxygen and hydroxyl groups on the surface of the substances have been widely used.[20-22] Antibacterial properties of metal Nanoparticles have found applications in various fields such as medical instruments and devices, water treatment and food processing. Antibacterial effect of copper Nanoparticles was reported by Yoon et al.[18, 23] Reported antifungal and bacteriostatic properties of copper Nanoparticles.[18, 24]

In this study synthesis of Nano copper and surface modification of the polyester fabric is carried out in one step. The role of glucose as stabilizer in this method and thus Copper sulfate reactions with sodium hydroxide at presence of glucose resulted in creating Copper Nano plates and their deposition on the fabric surface.

\section{MATERIAL AND METHODS}

The applied materials were obtained as follows: polyester fabrics with $165 \mathrm{~g} / \mathrm{m} 2$ from Hijab Co (Iran). Copper sulfate (CuSO4*5H2O) and Sodium hydroxide $(\mathrm{NaOH})$ and glucose has been prepared from Merck Co (Germany) were used. Absorption rate of Nano copper was studied by a spectrometer called Varian, Cary100 UV-VisNIR. Reflection spectra of Nano copper on the fabrics were investigated by a Spectrophotometer Color-Eye 7000A (USA) and 
antibacterial test. Surface morphology of the fabric was observed by scanning electron microscope TESCAN VEGA $5130 \mathrm{~mm}$ SEM/EDX (Britain). To determine chemical groups on the fabric, FT-IR spectrometer (model Bomemmb100) was (Germany) used. Also, crystal structure of the fabric was investigated by an XRay diffraction system (model XRD PTS 300) from SEIFERT Co (Germany). Tensile properties of fabric measured Testometric M500-25CT (UK). The accuracy synthesis of copper Nano plates in both. Then, Polyester fabric samples were prepared at $10^{*} 10 \mathrm{~cm} 2$ and washed using standard method in a washing bath including a non-ionic detergent $1 \mathrm{~g} / \mathrm{L}$ and sodium carbonate $1 \mathrm{~g} / \mathrm{L}$ at $60^{\circ} \mathrm{C}$ for $20 \mathrm{~min}$. The samples were washed and then dried. The main step which included Nano copper synthesis bath was performed by impregnation of the sample in the bath containing copper sulfate $0.05 \%$, sodium hydroxide and glucose $0.01 \%$ at $100{ }^{\circ} \mathrm{C}$ for 120 $\mathrm{min}$. The samples were then taken out and dried at $100^{\circ} \mathrm{C}$ for $4 \mathrm{~min}$ followed by curing at $150^{\circ} \mathrm{C}$ for $3 \mathrm{~min}$. The finished fabrics were washed and their properties were investigated.

\section{RESULTS}

\subsection{Synthesis of Nano copper in solution}

The copper Nano plates synthesized by bath containing copper sulfate, polyester fabric, sodium hydroxide, glucose as reducing agent. Therefore, in the method, synthesis of copper Nano plates and deposition onto the fabric in one-steps. Copper Nano plates were formed and physically attached to the polyester fabrics. The mechanism of reaction of copper sulfate with sodium hydroxide and glucose as reducing agent has been shown in Reaction. This can be done through the chemical and physical reaction that leads to formation of fabrics.

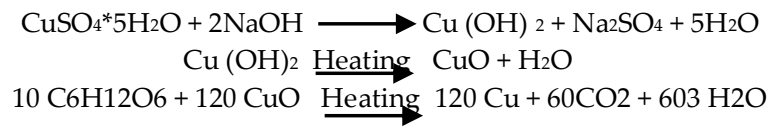

\subsection{Synthesis of Nano copper on polyester fabric}

In situ synthesis, the conversion $\mathrm{Cu}^{+}$to $\mathrm{CuO}$ is possibly by reducing agents and substitution of $\mathrm{Cu}+$ with $\mathrm{H}^{+}$can be due to the higher positive chemical potential of $\mathrm{Cu}^{+}(+0.34)$. Also Copper Nano plates were physically linked on the compact structure of the polyester fabric (Fig.1). By initiation of Nano copper synthesis on polyester fabric, they Grown and particle size have been bigger.

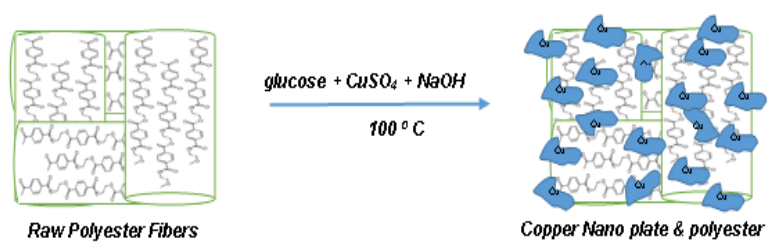

Fig. 1. Polyester fabric finished with copper Nano plate

\subsection{Absorption spectrometry}

In this experiment, the remaining solution of the treated samples was separated and analyzed using a Cary 100 (Fig.2), which revealed a peak around $600-650 \mathrm{~nm}$ that confirmed synthesis of

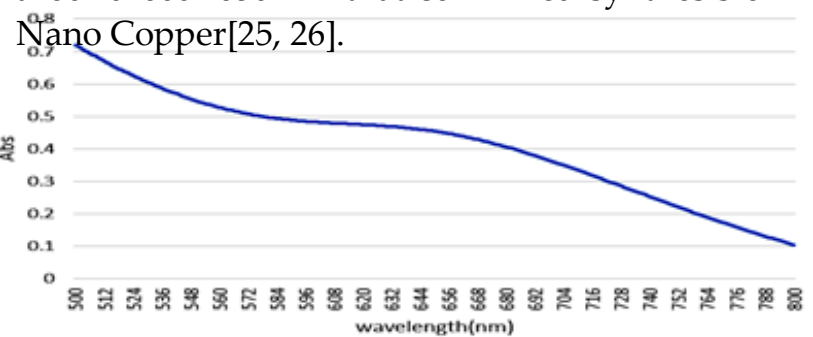

Fig. 2. Absorption spectrum of the remaining solution

\subsection{Reflection spectrometry}

The treated polyester fabrics with Nano copper and raw samples were analyzed using reflective spectrometer (Fig.3), through which the amount of $\Delta \mathrm{E}$ was calculated. Also, a significant peak was observed in the range 550-650 nm, which was not present in the raw samples, indicating the presence of copper Nano plates on the surface of the fabrics[27]. 
Also, copper diffusion into the polyester samples and reducing agent caused changes in the color of polyester samples. The changes of color indicated variations ranging from 1.8 to 3.6. Thus, the processing conditions were influenced on the color changes. Mild conditions caused low changes in color and hard conditions produced bigger changes in polyester color. This parameter should be optimized with other parameters such as tensile and others.

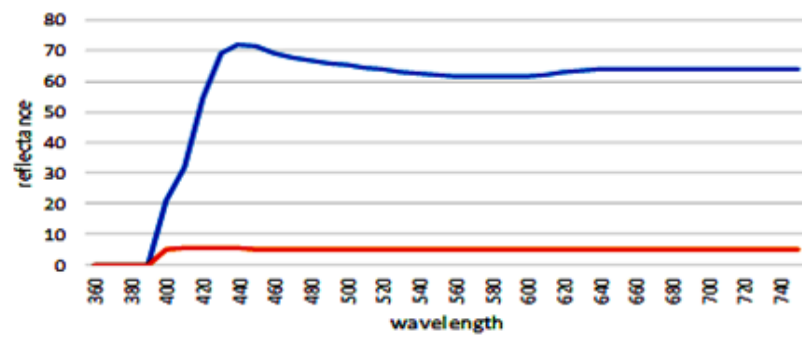

Fig. 3. Reflectance spectroscopy of the samples modified and raw

\subsection{X-ray diffraction (XRD)}

XRD is a simple instrument to determine unit cell size and shape for each combination. Diffraction methods are useful for qualitative analysis (phase identification), quantitative analysis (determining lattice parameter and analyzing phase fraction) and diffraction pattern (Fig. 4)[25].

The XRD peak positions were consistent with metallic copper. Sharp peaks of the XRD pattern indicated the crystalline nature. The peaks at $43.38^{\circ}, 50.44^{\circ}$ and $74.125^{\circ}$ corresponding to the Miller indices (111), (200) and (220), respectively, represented face centered cubic structure of copper. Lattice constant of the unit cell was $\mathrm{a}=3.615 \AA$ and its volume was $4.7245 \times 10^{-29}$ $\mathrm{m}^{-3}[18]$.

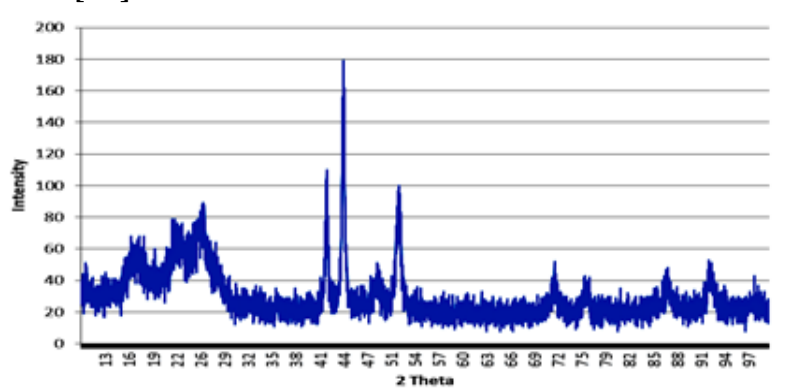

Fig. 4. X-ray diffraction of polyester fabrics modified
The synthesized Nano plates were found to be phase-pure copper without any impurity phase such as $\mathrm{CuO}, \mathrm{Cu}_{2} \mathrm{O}$ and $\mathrm{Cu}(\mathrm{OH})_{2}$. In order to do this, the treated sample was placed using $\mathrm{Kv} / 30$ $\mathrm{mA}$ data of 2 theta from 10 to 100 degrees and amount of 2 theta was found as shown in Table $1[18]$.

Table 1. 2 theta of synthesized copper Nano plates on polyester fabric

\begin{tabular}{ccc}
\hline 2theta & Int & $\mathrm{d}\left(\mathbf{A}^{\circ}\right)$ \\
\hline 44.36 & 179 & 2.0880 \\
51.6 & 100 & 2.5118 \\
75.88 & 34 & 1.4779 \\
\hline
\end{tabular}

Their crystal structure was analyzed by XRD using $\mathrm{Cu}-\mathrm{K} \alpha$ ray $(\gamma=0.154 \mathrm{~nm})$ within the range of 10 to 100 degree angle at 0.02 degrees in each registered step. The particles' average size was estimated by calculating width of the peaks created in the samples by means of sheerer formula: $\quad D=0.9 \lambda / \beta \cos \theta$

(Where $\beta=$ peak's width at half of maximum, $\lambda=$ length of X-ray radiation, $\theta=$ angle between radiations' open ray and radiation, $\mathrm{d}=$ particles' size).

In the SEM Figure such as sheerer formula observed copper Nano-plates on the fiber surface with thickness of 30-80 $\mathrm{nm}$.

\subsection{FTIR}

Infrared spectroscopy is done based on absorption of radiation and evaluation of vibrational mutation of multi atomic molecules and ions. This method is used mainly for detecting organic compounds because spectra of these compounds are usually complex and have a minimum and maximum number of peaks that can be used for the purposes of convention. FTIR in wave number range of $500-4000 \mathrm{~cm}-1$ used to prove the effectiveness of the processing conditions on the molecular structure of the treated polyester samples[25]. 
Fig.5 shows the chemical groups present in the fabrics before and after modification. The changes observed in the peaks showed alteration in the fabric chemical structure through the synthesis of Nano copper. Standard IR Absorption Frequencies in table 2 is shown.[2830]. But, the synthesis of Nano copper on polyester caused changes in some peaks. Carbonyl groups peaks of samples were shifted between 10 and $20 \mathrm{~cm}^{-1}$ and some of ester groups peaks shifted depending on the experiment conditions. The changes can be related to the types of reducing agent and processing conditions. Therefore, the shift in peaks indicated Nano copper synthesis changes due to the extension of polyester (Fig.5-a, 5-b).

Table 2. IR peaks identified by wave number peak location.[28-30]

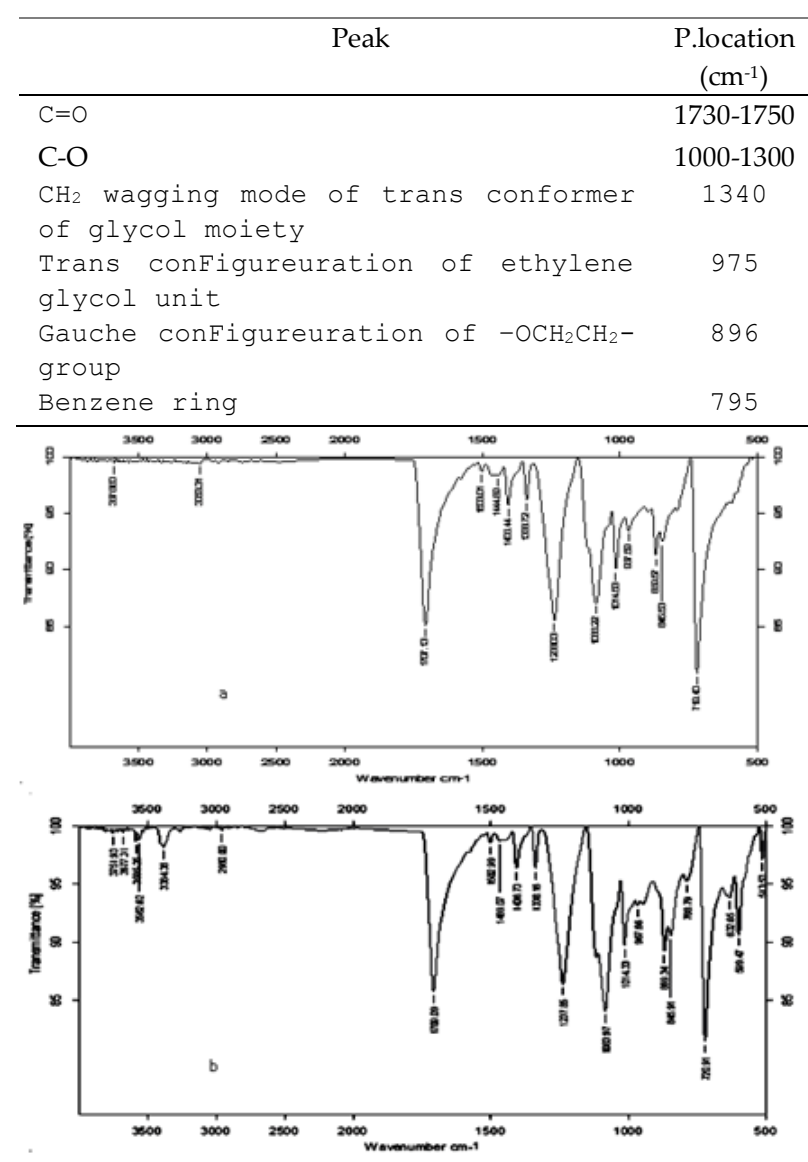

Fig. 5. FTIR spectrum of various fabrics: a) raw and b) modified with Copper Nano-plates

\subsection{SEM/EDX}

Surface morphology could be determined by means of electron microscope and analyzed by means of analyzer. Non-conductive materials are usually covered with a thin layer of carbon or gold for taking pictures. SEM proved synthesis and loading of copper Nano plates on the polyester fabrics as indicated in Fig.6. Nano materials were observed in the shape of Nanoplates on the fiber surface with thickness of 30-80 nm.
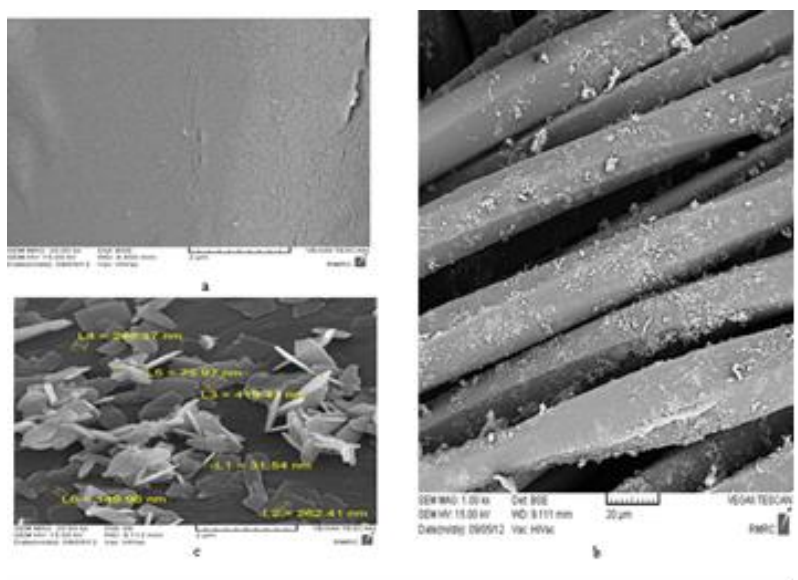

Fig. 6. SEM images of different fabric smples: a) raw with 20000X, b) finished with Nano copper 1000X, c) finished with Nano copper 20000X

The samples were analyzed by EDX to show elemental composition of the samples (Fig.7). EDX results presented in Table 3 that it confirmed the presence of Nano copper on the polyester fabrics and also proved the influences of the processing conditions on the copper content on the polyester surface. The copper Nano plate on the polyester fabrics surfaces in the range of $1.29 \%$ synthesized. The EDX results caused more synthesis of Nano copper on polyester fabrics.

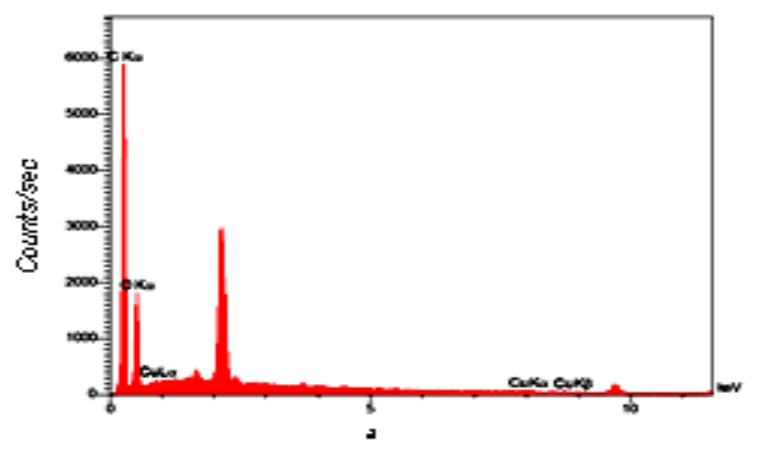




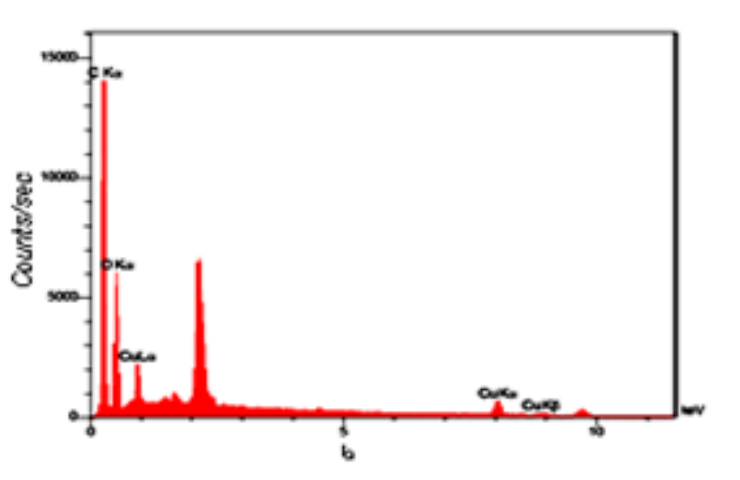

Fig. 7. EDX patterns of: a) raw and b) modified polyester with copper Nano plates

Table 3 shows atomic and weight percentages of different elements on the fabric sample. Only carbons and oxygen groups were observed on the raw samples which were related to the polyester groups; but, on the finished samples, copper In addition to carbon and hydrogen groups was found.

Table 3. Wt. \% and A\% elements on the polyester fabric of: a) raw, b) modified with copper Nano plates

\begin{tabular}{lccc}
\hline \multicolumn{3}{c}{ a. Raw sample } \\
\hline Elt & Line & $\mathrm{W} \%$ & $\mathrm{~A} \%$ \\
\hline $\mathrm{C}$ & $\mathrm{Ka}$ & 65.45 & 71.62 \\
$\mathrm{O}$ & $\mathrm{Ka}$ & 34.55 & 28.38 \\
$\mathrm{Cu}$ & $\mathrm{Ka}$ & 0.00 & 0.00 \\
& & 100.00 & 100.00 \\
\hline
\end{tabular}

\begin{tabular}{llll}
\hline & \multicolumn{3}{c}{ b. Modified sample } \\
\hline Elt & Line & W\% & A\% \\
\hline $\mathrm{C}$ & $\mathrm{Ka}$ & 58.20 & 67.97 \\
$\mathrm{O}$ & $\mathrm{Ka}$ & 34.76 & 30.48 \\
$\mathrm{Cu}$ & $\mathrm{Ka}$ & 7.04 & 1.55 \\
& & 100.00 & 100.00
\end{tabular}

\section{DISCUSSION}

This study showed successful treatment of polyester fabric with copper salt to synthesis copper Nano plates on the fabric surface to increase their applications. Also, this paper explored a new method of in situ synthesis of Nano copper within polyester fabrics. Availability and simplicity of the procedure were unique characteristics in this study. The synthesis of Nano copper confirmed by SEM, EDX and XRD patterns on polyester fabrics. Also the synthesis of Nano copper in the solution confirmed with UV visible absorption spectra. Further, the reasonable antibacterial properties indicated on the fine polyester fabrics on both $\mathrm{S}$. aurous and E. coli. And the Nano copper finishing agents could really kill the bacteria not just inhibit their growth. Overall, $6.48 \%$ enhance in tensile strength, 3.2 colour change and 2.35\% weight loss with good antibacterial properties. This study of glucose was used as a stabilizer agent. Thus copper sulfate reactions with sodium hydroxide in the presence of glucose at $100^{\circ} \mathrm{C}$ for 120 min resulted in creating copper Nano plates and their deposition on the fabric surface. The results indicated formation of copper Nano plates with thicknesses of 30 to $80 \mathrm{~nm}$ that mostly were physically link on the compact structure of the polyester fabric.

\section{References}

Tjong SC , Chen H (2004) Nanocrystalline materials and coatings. Materials Science and Engineering R 45:1-88

Chen D-H , Hsieha C-H (2002) Synthesis of Nickel Nanoparticlesin Aqueous Cationic Surfactant Solutions. Journal of Materials Chemistry - J MATER CHEM 12 (8):24122415

Riley MDK, Classen MDC, Stevens MLE, Burke MJP (1995) A large randomized clinical trial of a silver-impregnated urinary catheter: lack of efficacy and staphylococcal superinfection, . Am J Med 98:349-356

Crabtree JH, Burchette RJ, Siddiqi RA, Huen IT, Hadnott LL , Fishman A (2003) The efficacy of silver-ion implanted catheters in reducing 
peritoneal dialysisrelated infections. Perit Dial Int 23:368-374

Shan B, Cai Y-Z, Brooks JD, Corke H (2008) Antibacterial properties of Polygonum cuspidatum roots and their major bioactive constituents. Food Chem 109:530-537

Alt V, Bechert T, Steinrücke $\mathrm{P}$, Wagener $\mathrm{M}$, Seidel P, Dingeldein E, Domann E, Schnettler $R$ (2004) An in vitro assessment of the antibacterial properties and cytotoxicity of nanoparticulate silver bone cement. Biomaterials 25:4383-4391

Chang C-C, Lin C-K, Chan C-C, Hsu C-S , Chen C-Y (2006) Photocatalytic properties of nanocrystalline $\mathrm{TiO} 2$ thin film with $\mathrm{Ag}$ additions. Thin Solid Films 494:274-278

Dastjerdi R, Montazera M , Shahsavan S (2009) A new method to stabilize nanoparticles on textile surfaces. Colloids SurfA 345:202-210

Dastjerdi R, Mojtahedi MRM, Shoushtari AM , Khosroshahi A (2010) Investigating the production and properties of $\mathrm{Ag} / \mathrm{TiO} 2 / \mathrm{PP}$ antibacterial nanocomposite filament yarns. J Text Inst 101:204-213

Montazer M , Afjeh MG (2007) Simultaneous, Xlinking and antimicrobial finishing of cotton fabric. J Appl Polym Sci 103:178-185

hosseinkhani m, Montazer M, Eskandarnejad S, Rahimi Mk (2012) Simultaneous in situ synthesis of nano silver and wool fiber fineness enhancement using sulphur based reducing agents. Colloids and Surfaces A: Physicochemical and Engineering Aspects 415:431-438

Park BK, Jeong S, Kim D, Moon J, Lim S , Kim JS (2007) Synthesis and size control of monodisperse copper nanoparticles by polyol method. Journal of Colloid and Interface Science 311:417-424

Kumar RV, Mastai Y, Diamant Y, Gedanken A (2001) Sonochemical Synthesis of Amorphous $\mathrm{Cu}$ and Nanocrystalline $\mathrm{Cu} 2 \mathrm{O}$ Embedded in A Polyaniline Matrix. J MaterChemistry Letters 11 (1209-13)

Wu S-H , Chen D-H (2004) Synthesis of highconcentration $\mathrm{Cu}$ nanoparticles in aqueous CTAB solutions. J Colloid Interface Sci 273 (1):165-169
Dewan M, Kumar A, Saxena A, De A , Mozumdar S (2012) Using Hydrophilic Ionic Liquid, [bmim]BF4 - Ethylene Glycol System as a Novel Media for the Rapid Synthesis of Copper Nanoparticles. PLoS ONE 7(1): e29131. doi:10.1371/journal.

Khanna PK, More P, Jawalkar J, Patil Y, Rao NK (2009) Synthesis of Hydrophilic Copper Nanoparticles: Effect of Reaction Temperature. J Nanoparticle Res 11:793-799

Wei Y, Chen S, Kowalczyk B, Huda S, Gray TP , Grzybowski BA (2010) Synthesis of stable, low-dispersity copper nanoparticles and nanorods and their antifungal and catalytic properties. The Journal of Physical Chemistry C 114:15612-15616

Ramyadevi J, Jeyasubramanian K, Marikani A, Rajakumar G, Rahuman AA (2012) Synthesis and antimicrobial activity of copper nanoparticles. Materials Letters (71):114-116

Morones JR, Elechiguerra JL, Camacho A, Holt K, Kouri JB, Ramírez JT , Yacaman MJ (2005) The bactericidal effect of silver nanoparticles. Nanotechnology Nanotechnology 16 (10):2346-2353

Montazer M , Jolaei MM (2010) $\beta$-Cyclodextrin stabilized on three-dimensional polyester fabric with different crosslinking agents. Journal of Applied Polymer Science 116 (1):210-217. doi:10.1002

Montazer M , Jolaei MM (2010) Novel spacer three-dimensional polyester fabric with $\beta$ cyclodextrin and butane tetra carboxylic acid. Journal of Textile Institute 101 (2):165-172

Valle EMMD (2004) Review Cyclodextrins and their uses. JProcess Biochemistry 39:1033-1046

Yoon K-Y, Byeon JH, Park J-H , Hwang J (2007) Susceptibility constants of Escherichia coli and Bacillus subtilis to silver and copper nanoparticles. The Science of the total environment $\quad 373 \quad$ (2-3):572-575. doi:10.1016/j.scitotenv.2006.11.007

Cioffi N, Torsi L, Ditaranto N, Tantillo G, Ghibelli L, Sabbatini L, Bleve-Zacheo T, D'Alessio M, Zambonin PG , Traversa E (2005) Copper Nanoparticle/Polymer Composites with Antifungal and 
Bacteriostatic Properties. Chem Mater 17 (21):5255-5262

Rahman A, Ismail A, Jumbianti D, Magdalena S, Sudrajat H (2009) SYNTHESIS OF COPPER OXIDE NANO PARTICLES BY USING Phormidium cyanobacterium. Indo J Chem 9 (3):355 - 360

Yin M, Wu C-K, Lou Y, Burda C, Koberstein JT, Zhu Y, O'Brien S (2005) Copper oxide nanocrystals. Journal of the American Chemical Society 127 (26):9506-9511. doi:10.1021/ja050006u

Bol AA, Ferwerda J, Bergwerff JA, Meijerink A (2002) Luminescence of nanocrystalline ZnS:Cu2+. Journal of Luminescence 99 (4):325-334

Hobbs J-PA, Sung CSP, Krishnan K, Hill S (1983) Characterization of Surface Structure and Orientation in Polypropylene and Poly (ethylene terephthalate) Films by Modified Attenuated Total Reflection IR Dichroism Studies. Macromolecules 16 (2):193-199

Caba KDl, Guerrero P, Mondragon Ia , Kenny JM (1998) Comparative Study by DSC and FTIR Techniques of an Unsaturated Polyester Resin Cured at Different Temperatures. Polymer International 45 (4):333-338

Enlow EM, Kennedy JL, Nieuwland AA, Hendrix JE , Morgan SL (2005) Discrimination of Nylon Polymers Using Attenuated Total Reflection Mid-Infrared Spectra and Multivariate Statistical Techniques. Applied Spectroscopy 59 (8)

Akaike, H. (1973). Information theory and an extension of the maximum likelihood principle. Em: Proceedings of the 2nd International Symposium on Information Theory, pp. 267-281.

Ferrari, S. L. P., Cribari-Neto, F. (2004). Beta regression for modelling rates and proportions. Journal of Applied Statistics, 31(7), 799-815.

McCullagh, P., Nelder, J. (1989). Generalized linear models, 2o edn. Chapman and Hall.

R Development Core Team (2009). R: A language and environment for statistical computing. $\mathrm{R}$ Foundation for Statistical Computing, Vienna, Austria, URL 